\title{
Deconstructing Obstructive Defecation Syndrome with Adaptive Biofeedback
}

\author{
H. Heinrich ${ }^{1}$
}

Accepted: 2 June 2021 / Published online: 15 June 2021

(C) The Author(s), under exclusive licence to Springer Science+Business Media, LLC, part of Springer Nature 2021

The common syndromes of chronic constipation (CC) and obstructive defecation syndrome (ODS) have a significant impact on the quality-of-life of affected patients. In up to $40 \%$ of patients, CC is caused by the behavioral condition termed dyssynergic defecation (DD), which entails a paradoxical contraction, inadequate relaxation, or both of the puborectalis muscle, often associated with inadequate propulsive forces during defecation. Though the symptoms and physiological testing are the mainstay of diagnosis. [1, 2], the diagnosis of DD is however often difficult as more than half of healthy volunteers have manometric findings consistent with DD [3], possibly due to procedure-related factors such as positioning in the left lateral position and the embarrassing nature of the investigation [4]. According to several guidelines, DD can therefore only be diagnosed on the basis of at least two complementary tests (i.e., balloon expulsion tests, manometry, proctography, or defecography) $[4,5]$.

Biofeedback is an operant conditioning therapy; in the case of ODS it entails either visualization of anorectal and abdominal muscle activity with manometry or electromyography in order to help the patient increase intra-abdominal pressure and relax the anal sphincter musculature during defecation. Due to its proven efficacy in ODS in several randomized trials-showing it to be more effective than sham feedback or medical therapy with laxatives and benzodiazepines-it is recommended by several guidelines for the treatment of chronic constipation as a first-line treatment. Sustained symptom improvement can be achieved in more than $70 \%$ of patients [6-8].

Patient selection for biofeedback is crucial as, especially patients with constipation due to other causes do not benefit to the same degree as do patients with ODS [9]. Further

\footnotetext{
H. Heinrich

Henriette.Heinrich@usz.ch

$1 \quad$ Stadtspital Waid und Triemli, Department of Gastroenterology, University of Zürich, Zurich, Switzerland
}

predictors of success are the patient's motivation and adherence to treatment, raised symptom severity, and digital assistance with stooling $[10,11]$. Another obstacle for effective biofeedback training is the availability of specialist biofeedback therapists. Since office-based biofeedback therapy is expensive and time consuming, randomized controlled trials have recently shown promising results for home-based biofeedback devices in order to provide cost-effective treatment to more patients [12].

In the current issue of Digestive Diseases and Sciences, Yuemei $\mathrm{Xu}$ and colleagues present the interesting result of a randomized controlled trial of adaptive versus fixed (i.e., conservative) biofeedback therapy in 42 patients with constipation due to CC [13]. Regardless of randomization, all patients received two weeks of sham biofeedback. The adaptive biofeedback regimen (ABF) included visual, auditive and qualitative feedback throughout the sessions and more importantly thresholds (i.e., sensation) were adapted specifically to the patients' baseline characteristics and abilities and were furthermore adapted according to achieved goals. During the Fixed Biofeedback Regimen (FBR), the experimental design was similar with the exception that the thresholds and goals were fixed and not individualized for the patient. Both groups were asked to perform exercises at home and attended biweekly training sessions. Treatment success was defined by improved physiological results, symptom improvement, need for medication, and the number of complete spontaneous bowel movements per week, as is recommended by the US Food and Drug Administration for trials of medications in chronic constipation.

Twenty-one patients with $\mathrm{CC}$ completed $\mathrm{ABF}$ and 21 underwent FBR. The number of complete bowel movements, symptoms of constipation, need for medications, and physiological results were significantly improved in the ABF group when compared with patients who underwent FBR, clearly demonstrating the efficacy of a tailored biofeedback treatment individualized to the patients' needs and baseline criteria. 
As in many other biofeedback studies, this study can be seen as partly biased as there was no full sham intervention since blinding was not possible. Another criticism is the small number of patients included in the study as differences in bowel movements and physiologic parameters between the two groups might be overestimated due to the low number of patients. Nevertheless, the data generated by this study provide a compelling argument for individualized tailored feedback regimens that can be investigated in larger randomized controlled trials in order to improve patient outcomes and further the current success and popularity of biofeedback treatment. It remains to be seen if a tailored biofeedback approach might also be of benefit in refractory cases of chronic constipation due to DD.

\section{References}

1. Bharucha AE, Lacy BE. Mechanisms, evaluation, and management of chronic constipation. Gastroenterology 2020;158:123249.e3.

2. Skardoon GR, Khera AJ, Emmanuel AV, Burgell RE. Review article: dyssynergic defaecation and biofeedback therapy in the pathophysiology and management of functional constipation. Aliment Pharmacol Ther 2017;46:410-23.

3. Grossi U, Carrington EV, Bharucha AE, Horrocks EJ, Scott SM, Knowles CH. Diagnostic accuracy study of anorectal manometry for diagnosis of dyssynergic defecation. Gut 2016;65:447-55.

4. Carrington EV, Scott SM, Bharucha A, Mion F, Remes-Troche JM, Malcolm A et al. Expert consensus document: advances in the evaluation of anorectal function. Nat Rev Gastroenterol Hepatol 2018;15:309-23.

5. Carrington EV, Heinrich $\mathrm{H}$, Knowles $\mathrm{CH}$, Fox M, Rao S, Altomare DF et al. The international anorectal physiology working group
(IAPWG) recommendations: standardized testing protocol and the London classification for disorders of anorectal function. Neurogastroenterol Motil. 2020;32:e13679.

6. Chiarioni G. Biofeedback treatment of chronic constipation: myths and misconceptions. Tech Coloproctol 2016;20:611-8.

7. Rao SS, Benninga MA, Bharucha AE, Chiarioni G, Di Lorenzo C, Whitehead WE. ANMS-ESNM position paper and consensus guidelines on biofeedback therapy for anorectal disorders. Neurogastroenterol Motil 2015;27:594-609.

8. Serra J, Pohl D, Azpiroz F, Chiarioni G, Ducrotté P, Gourcerol $\mathrm{G}$ et al. European society of neurogastroenterology and motility guidelines on functional constipation in adults. Neurogastroenterol Motil. 2020;32:e13762.

9. Chiarioni G, Salandini L, Whitehead WE. Biofeedback benefits only patients with outlet dysfunction, not patients with isolated slow transit constipation. Gastroenterology 2005;129:86-97.

10. Patcharatrakul T, Valestin J, Schmeltz A, Schulze K, Rao SSC. Factors associated with response to biofeedback therapy for dyssynergic defecation. Clin Gastroenterol Hepatol 2018;16:715-21.

11. Heinrich H, Fruehauf H, Sauter M, Steingotter A, Fried M, Schwizer W, et al. The effect of standard compared to enhanced instruction and verbal feedback on anorectal manometry measurements. Neurogastroenterol Motil. 2013;25:230-7, e163.

12. Rao SSC, Valestin JA, Xiang X, Hamdy S, Bradley CS, Zimmerman MB. Home-based versus office-based biofeedback therapy for constipation with dyssynergic defecation: a randomised controlled trial. Lancet Gastroenterol Hepatol 2018;3:768-77.

13. Xu Y, Li X, Xia F, Xu F, Chen JDZ. Efficacy of a modified training program of adaptive biofeedback therapy for dyssynergic defecation in patients with chronic constipation. Dig Dis Sci. (Epub ahead of print). https://doi.org/10.1007/s10620-021-07094-z.

Publisher's Note Springer Nature remains neutral with regard to jurisdictional claims in published maps and institutional affiliations. 